ITP-SB-91-53

IASSNS-HEP-91/68

October 1991

\title{
Duality, Quotients, and Currents
}

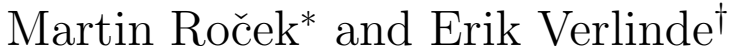 \\ School of Natural Sciences \\ Institute for Advanced Study \\ Princeton, NJ 08540
}

\begin{abstract}
We study the generalization of $R \rightarrow 1 / R$ duality to arbitrary conformally invariant sigma models with an isometry. We show that any pair of dual sigma models can be represented as quotients of a self-dual sigma model obtained by gauging different combinations of chiral currents. This observation is used to clarify the interpretation of the generalized duality as a symmetry of conformal field theory. We extend these results to $N=2$ supersymmetric sigma models.
\end{abstract}

* Permanent address: ITP, SUNY at Stony Brook, Stony Brook NY 11794-3840.

Email: rocek@dirac.physics.sunysb.edu

$\dagger$ Email: verlinde@iassns.bitnet 


\section{Introduction}

It is known from prehistoric times that the vacuum Maxwell equations are preserved under the interchange of the field strength $F_{\mu \nu}=\partial_{\mu} A_{\nu}-\partial_{\nu} A_{\mu}$ with its dual $\frac{1}{2} \epsilon^{\mu \nu \rho \sigma} F_{\rho \sigma}$. This duality transformation exchanges the electric and magnetic fields, and at the same time, the field equations $\partial^{\mu} F_{\mu \nu}=0$ with the Bianchi identities $\epsilon^{\mu \nu \rho \sigma} \partial_{\nu} F_{\rho \sigma}=0$. In two dimensions a similar duality transformation can be defined to act on the "field strength" of a scalar field $P_{\mu}=\partial_{\mu} \phi$ :

$$
P_{\mu} \leftrightarrow \epsilon^{\mu \nu} P_{\nu}
$$

Again, this exchanges the field equations $\partial^{\mu} P_{\mu}=0$ with the Bianchi identities $\epsilon^{\mu \nu} \partial_{\mu} P_{\nu}=0$.

More generally, in any two-dimensional nonlinear sigma model with an isometry, one may define a duality transformation as follows: One gauges the isometry, and adds to the action a Lagrange multiplier that constrains the gauge field to be flat, i.e., constrains its field strength to vanish. This generalizes the well-known $R \rightarrow 1 / R$ duality [1]. In this paper we clarify the interpretation of this generalized duality in terms of conformal field theory. In particular, we show that any dual pair can be obtained by gauging different combinations of chiral currents in a higher dimensional sigma model, and extend these results to $N=2$ superspace.

The plan of the paper is as follows: First we review duality and discuss when it is an exact equivalence. In section 3 we discuss isometries and the geometric significance of chiral currents. Our main result is derived in subsection 3.2: we interpret duality in terms conformal field theory quotients by chiral currents. This is used to complete the discussion of duality as a symmetry of conformal field theories. We end section 3 with some general remarks on duality. In section 4, we extend the discussion to $N=2$ supersymmetric sigma models. After reviewing superspace kinematics, in subsection 4.2 we discuss axial and vector gauge fields and explain how they are used to implement $N=2$ quotients and duality transformations. We then review the explicit form of duality in $N=2$ superspace, and present the construction of dual pairs as quotients. Finally, we show how $N=2$ duality works in components. In an appendix, we have added a brief description of the geometry of $N=2$ quotients. 


\section{Duality in non-linear sigma models}

We consider the sigma model action

$$
S=\frac{1}{2 \pi} \int d^{2} z\left(g_{i j}+b_{i j}\right) \partial x^{i} \bar{\partial} x^{j}
$$

where $g$ is the metric tensor on some manifold and $b$ is a potential for the torsion 3 -form $T=\frac{3}{2} d b$. This action is invariant under the isometry

$$
\delta x^{i}=\epsilon \mathbf{k}^{i}
$$

when the vector field $\mathbf{k}^{i}$ satisfies Killing's equation $\mathcal{L}_{\mathbf{k}} g_{i j}=\mathbf{k}_{i ; j}+\mathbf{k}_{j ; i}=0$ and in addition $\mathcal{L}_{\mathbf{k}} T=0$; the latter implies that locally

$$
\mathcal{L}_{\mathbf{k}} b=d \omega .
$$

for some 1 -form $\omega$. Here $\mathcal{L}_{\mathbf{k}}$ and $d$ denote the space-time Lie- and exterior derivative. For sigma models with a dilaton term

$$
S_{d i l}=\frac{1}{2 \pi} \int d^{2} z \Phi R^{(2)}
$$

the dilaton field $\Phi$ must satisfy $\mathbf{k}^{i} \Phi_{, i}=0$. One can now choose coordinates $\left\{x^{i}\right\}=$ $\left\{x^{0}, x^{a}\right\}$ such that the isometry acts by translation of $x^{0} \equiv \theta$, and all fields $g, b$ and $\Phi$ are independent of $\theta$. (Here we used the fact that the 2 -form $b$ is defined only up to (space-time) gauge transformations $b \rightarrow b+d \lambda$, for some 1 -form $\lambda$; the 1 -form $\omega$ defined in (2.3) transforms under this gauge symmetry as $\omega \rightarrow \omega+\mathcal{L}_{\mathbf{k}} \lambda$.)

The dual theory can be found from the first order action $S_{1}[2$, 3, 4]

$$
\begin{aligned}
S_{1}=\frac{1}{2 \pi} \int d^{2} z\left[g_{00} A \bar{A}\right. & +\left(g_{0 a}+b_{0 a}\right) A \bar{\partial} x^{a}+\left(g_{a 0}+b_{a 0}\right) \partial x^{a} \bar{A} \\
& \left.+\left(g_{a b}+b_{a b}\right) \partial x^{a} \bar{\partial} x^{b}+\tilde{\theta}(\partial \bar{A}-\bar{\partial} A)\right]
\end{aligned}
$$

If one integrates out the Lagrange multiplier field $\tilde{\theta}$, on a topologically trivial worldsheet the gauge fields are pure gauge, $A=\partial \theta, \bar{A}=\bar{\partial} \theta$, and one recovers the original model (2.1). Classically, if one integrates out the gauge fields $A, \bar{A}$ one finds the dual model; this is a new theory with action $\tilde{S}$ 田, 5

$$
\tilde{S}=\frac{1}{2 \pi} \int d^{2} z\left(\tilde{g}_{i j}+\tilde{b}_{i j}\right) \partial \tilde{x}^{i} \bar{\partial} \tilde{x}^{j},
$$


where $\left\{\tilde{x}^{i}\right\}=\left\{\tilde{\theta}, x^{a}\right\}$ and

$$
\begin{array}{ll}
\tilde{g}_{00}=\frac{1}{g_{00}}, & \tilde{g}_{0 a}=\frac{b_{0 a}}{g_{00}}, \quad \tilde{g}_{a b}=g_{a b}-\frac{g_{a 0} g_{0 b}+b_{a 0} b_{0 b}}{g_{00}} \\
\tilde{b}_{0 a}=\frac{g_{0 a}}{g_{00}}, & \tilde{b}_{a b}=b_{a b}+\frac{g_{a 0} b_{0 b}+b_{a 0} g_{0 b}}{g_{00}} .
\end{array}
$$

Quantum mechanically, the duality transformation receives corrections from the jacobian that comes from integrating out the gauge fields; at one loop, this leads to a simple shift in the dilaton [6, 4, 5]:用

$$
\Phi \rightarrow \Phi+\ln g_{00}
$$

With this shift, if the original theory was conformally invariant, at least to one-loop order, the dual theory will be as well. Note that in general, the geometry of the sigma model is completely changed by the duality transformation.

It is natural to ask if the dual models are truly equivalent as conformal field theories. We first consider global issues of the procedure. Let us assume that the isometry corresponds to a compact $U(1)$-group, so that the coordinate $\theta$ is periodic with period $2 \pi$. The constraint on $A, \bar{A}$ that comes from integrating out the Lagrange multiplier $\tilde{\theta}$ implies $A, \bar{A}$ are flat, but in principle they still may have nontrivial holonomies around non-contractible loops. In general, this would mean that the dual theory will have twisted sectors, and the duality transformation should then be thought of as an orbifold construction. However, by giving $\tilde{\theta}$ the appropriate periodicity, the holonomies are also constrained to vanish: the resulting winding modes of $\tilde{\theta}$ act as Lagrange multipliers for the holonomies. For example, for a worldsheet with the topology of a torus the action (2.5) contains the term

$$
S_{w i n d}=n_{a} \oint_{b} A+n_{b} \oint_{a} A
$$

where $n_{a}$ and $n_{b}$ are the winding numbers of $\tilde{\theta}$ around the $a$ - and $b$-cycle on the torus. The sum over these winding numbers produces a periodic delta-function for

*For some recent results on higher order effects, see [7]

† We thank E. Witten for pointing this out to us. 
the holonomy, which by tuning the periodicity makes the holonomy trivial as an element of $U(1)$. Explicitly, we find that $\tilde{\theta}$ should also have period $2 \pi$. Notice that this is consistent with the fact that for a $U(1)$ gauge field the integrated curvature $\int F$ takes only integer values: the integral over the periodic constant mode of $\tilde{\theta}$ precisely yields the required Kronecker delta (not a Dirac delta) to put $\int F$ to zero. Thus we may conclude that the action (2.5) indeed is equivalent to the original model without the gauge field.

\section{Duality and Quotients}

The second step in the duality transformation is integrating out the gauge field; this does not have an obvious interpretation in terms of conformal field theory because the current associated with the isometry in general does not correspond to a chiral current, i.e., to a Kač-Moody type current algebra. In this section, we show how the general (abelian) duality transformations can be reformulated as a construction that does involve chiral currents. This construction will allow us to complete the proof that duality is a true symmetry of conformal field theory.

\subsection{ISOMETRIES AND CHIRAL CURRENTS}

As a preparation we investigate the following question: when do isometries lead to chiral currents? We find that the Noether current associated with the isometry (2.2) is, in general coordinates, given by

$$
\begin{aligned}
& J=\mathbf{k}^{i}\left(g_{i j}-b_{i j}\right) \partial x^{j}+\omega_{j} \partial x^{j} \\
& \bar{J}=\mathbf{k}^{i}\left(g_{i j}+b_{i j}\right) \bar{\partial} x^{j}-\omega_{j} \bar{\partial} x^{j}
\end{aligned}
$$

where $\omega$ is the 1 -form that appeared in (2.3). It is easily shown using the equations of motion that this current is conserved: $\partial \bar{J}+\bar{\partial} J=0$. However, in general the chiral components $J$ and $\bar{J}$ are not conserved separately, as is illustrated by the familiar example of the Lorentz generators.

To have a chiral current $J$, satisfying $\bar{\partial} J=0$, we must be able to find a gauge for $b$ such that $\bar{J}=0$. From (3.1) we see that this implies that $\omega_{j}=\mathbf{k}^{i}\left(g_{i j}+b_{i j}\right)$. Inserting 
this into (2.3) gives a more restrictive condition on the $b$-field, which combined with Killing's equation implies that the vector $\mathbf{k}^{i}$ must be covariantly constant with respect to the connection $D_{i}^{+}$

$$
D_{i}^{+} \mathbf{k}_{j}=\partial_{i} \mathbf{k}_{j}-\Gamma_{i j}^{k} \mathbf{k}_{k}-T_{i j}^{k} \mathbf{k}_{k}=0
$$

where $T$ is the torsion tensor. At this point there are two possibilities:

$$
\text { (i) } T_{i j}^{k} \mathbf{k}_{k}=0, \quad \text { or } \quad \text { (ii) } T_{i j}^{k} \mathbf{k}_{k} \neq 0 \text {. }
$$

In the first case the condition (3.2) implies that the coordinate $\theta$ corresponding to the Killing vector represents a free scalar that decouples from the rest of the theory. Possibility (ii) is more interesting and leads to an action of the form

$$
S_{L}=\frac{1}{2 \pi} \int d^{2} z\left[\partial \theta \bar{\partial} \theta+G_{a}(x) \partial x^{a} \bar{\partial} \theta\right]+S[x]
$$

Clearly this action gives rise to a (left) chiral current $J^{L}=\partial \theta+\frac{1}{2} G_{a} \partial x^{a}$ that generates the chiral symmetry $\delta \theta=\alpha(z)$.

A somewhat curious property of this type of action is that by applying the duality transformation (2.7) we get back the same action, i.e.,

$$
g_{i j}=\tilde{g}_{i j}, \quad b_{i j}=\tilde{b}_{i j}
$$

In this sense a sigma model with a chiral current can be called self-dual,f although one can consider more general self-dual models for which (3.4) holds up to diffeomorphisms.

\subsection{Duality And Quotients By Chiral CURREnts}

Even though the current that generates the isometry is in general not chiral, the corresponding duality transformation can be understood in terms of chiral currents. The basic clue comes from a interesting recent example: the stringy two dimensional black hole [8 constructed as a quotient of the $S l(2, \mathbb{R})$ WZW model by a $U(1)$

\footnotetext{
*To really make it self-dual one should adjust the periodicity of $\theta$ to its self-dual value.
} 
subgroup. In [9, 10, 11, it was shown that two different quotients (resulting from axial vs. vector gaugings) gave equivalent CFT's with geometries dual in the sense discussed above. We now generalize this construction to any pair of dual manifolds with dimension $d$.

We start with a $d+1$ dimensional sigma model which in addition to the chiral current $J^{L}$ has a right-handed chiral current $\bar{J}^{R}$. From the above discussion we see that this requires a second isometry with a covariantly constant Killing vector with respect to the connection $D_{i}^{-}$, which differs from $D_{i}^{+}$in the sign of the torsion term. The most general action then takes the form!

$S_{L R}=\frac{1}{2 \pi} \int d^{2} z\left[\partial \theta_{L} \bar{\partial} \theta_{L}+\partial \theta_{R} \bar{\partial} \theta_{R}+2 B(x) \partial \theta_{R} \bar{\partial} \theta_{L}+G_{a}^{R} \partial \theta_{R} \bar{\partial} x^{a}+G_{a}^{L} \partial x^{a} \bar{\partial} \theta_{L}\right]+S[x]$

where $x^{a}$ are coordinates on an arbitrary manifold with an arbitrary WZW term, i.e., $S[x]=\frac{1}{2 \pi} \int d^{2} z\left(G_{a b}+B_{a b}\right) \partial x^{a} \bar{\partial} x^{b}$. This action has separately conserved left and right handed currents

$$
J^{L}=\partial \theta_{L}+B \partial \theta_{R}+\frac{1}{2} G_{a}^{L} \partial x^{a} ; \quad \bar{J}^{R}=\bar{\partial} \theta_{R}+B \bar{\partial} \theta_{L}+\frac{1}{2} G_{a}^{R} \bar{\partial} x^{a}
$$

corresponding to the $U(1)_{R} \times U(1)_{L}$ symmetry $\delta \theta_{R}=\alpha_{R}(\bar{z}), \delta \theta_{L}=\alpha_{L}(z)$. Next we can choose to gauge an axial or vector subgroup

$$
\delta_{V, A} \theta_{R}=\alpha, \quad \delta_{V, A} \theta_{L}= \pm \alpha
$$

For the gauged action we take the "minimally coupled" action that follows from (3.5) by the prescription

$$
\begin{array}{rlrl}
\partial \theta_{R} & \rightarrow \partial \theta_{R}+\frac{1}{2} A, & \partial \theta_{L} & \rightarrow \partial \theta_{L} \pm \frac{1}{2} A \\
\bar{\partial} \theta_{R} \rightarrow \bar{\partial} \theta_{R}+\frac{1}{2} \bar{A}, & \bar{\partial} \theta_{L} \rightarrow \bar{\partial} \theta_{L} \pm \frac{1}{2} \bar{A}
\end{array}
$$

and add the gauge invariant term

$$
\frac{1}{2}\left(\theta_{R} \mp \theta_{L}\right)(\partial \bar{A}-\bar{\partial} A)
$$

\footnotetext{
${ }^{\dagger}$ The action for the $S L(2, \mathbb{R})$ WZW-model is of the form 3.5$)$ with $G_{a}^{R}=G_{a}^{L}=0, B(x)=$ $\cosh (x)$, and $S[x]=-\frac{1}{2 \pi} \int d^{2} z \partial x \bar{\partial} x$.
} 
We include this term so that the gauge-field couples directly to chiral currents $J^{L}$ and $\bar{J}^{R}$ in the gauged action:

$$
S_{\text {gauged }}^{ \pm}=S_{L R}+\frac{1}{2 \pi} \int d^{2} z\left[A \bar{J}^{R} \pm \bar{A} J^{L}+\frac{1}{2} A \bar{A}(1 \pm B)\right]
$$

This ensures that when the sigma-model described by (3.5) is conformally invariant, the same holds for the gauged models (and vice versa). We come back to this point below.

Eliminating the gauge field from (3.10) gives

$S_{V}=\frac{1}{2 \pi} \int d^{2} z\left[\left(\frac{1+B}{1-B}\right) \partial \tilde{\theta} \bar{\partial} \tilde{\theta}+\frac{G_{a}^{R}}{1-B} \partial \tilde{\theta} \bar{\partial} x^{a}+\frac{G_{a}^{L}}{1-B} \partial x^{a} \bar{\partial} \tilde{\theta}+\frac{1}{2} \frac{G_{a}^{L} G_{b}^{R}}{1-B} \partial x^{a} \bar{\partial} x^{b}\right]+S[x]$

for the vector gauging, and

$S_{A}=\frac{1}{2 \pi} \int d^{2} z\left[\left(\frac{1-B}{1+B}\right) \partial \theta \bar{\partial} \theta+\frac{G_{a}^{R}}{1+B} \partial \theta \bar{\partial} x^{a}-\frac{G_{a}^{L}}{1+B} \partial x^{a} \bar{\partial} \theta-\frac{1}{2} \frac{G_{a}^{L} G_{b}^{R}}{1+B} \partial x^{a} \bar{\partial} x^{b}\right]+S[x]$

for the axial gauging, where

$$
\theta=\theta_{R}-\theta_{L}, \quad \tilde{\theta}=\theta_{R}+\theta_{L}
$$

We see that these actions describe different sigma models, each with an isometry corresponding to translations in $\theta$ and $\tilde{\theta}$ respectively. If we choose

$$
\frac{1+B}{1-B}=g_{00}, \quad \frac{G_{a}^{R, L}}{1-B}=g_{0 a} \pm b_{0 a}, \quad G_{a b}+B_{a b}+\frac{1}{2} \frac{G_{a}^{L} G_{b}^{R}}{1-B}=g_{a b}+b_{a b}
$$

we find that (3.11) and (3.12) are precisely related via the duality transformation (2.7). By taking into account the jacobian that arises from integrating out the gauge field, we find that the dilaton field gets shifted by

$$
\Phi \rightarrow \Phi+\ln (1 \pm B)
$$

which is also in accordance with (2.8). It follows that any pair of dual sigma models can be obtained in this way. 
As mentioned above, the action (3.5) with chiral currents is conformally invariant if the dual pair (3.11-3.12) are. One can explicitly check that this is true at one-loop order. This is most easily done using the previous observation that duality respects the one-loop beta functions. We start with the gauged action (3.10) and add a Lagrange multiplier term

$$
\frac{1}{2 \pi} \int d^{2} z \phi(\partial \bar{A}-\bar{\partial} A) .
$$

Note that for duality transformations, it doesn't matter whether we use "minimal coupling" (3.8) or the gauging (3.10), as terms such as (3.9) can be absorbed into the Lagrange multiplier term. We find that the dual of (3.10) with respect to the vector (axial) symmetry is (after shifting $\theta \rightarrow \theta+\phi$ ) precisely the action $S_{V}\left(S_{A}\right)$ of (3.11,3.12), respectively, with an extra, decoupled free field represented by $\phi$. This makes clear that when the one-loop $\beta$-functions vanish for one of the three actions $S_{A}, S_{V}$ and $S_{L R}$, the same holds for the other two.

We can now use the above quotient construction to fully establish that the dual sigma models are equivalent as conformal field theories as follows: First we note that the two actions (3.11) and (3.12) are simply related by reversing the sign of $B$ and $G_{a}^{L}$. This operation is a symmetry of the original action (3.5) in the sense that it can be undone by the coordinate transformation $\theta_{L} \rightarrow-\theta_{L}$. The net effect of the combined operation, however, is that the vector and axial gaugings are interchanged. From this we may conclude that these two gaugings are, at least locally, equivalent. To extend this to a global equivalence we need to impose that $\theta=\theta_{R}-\theta_{L}$ and $\tilde{\theta}=\theta_{R}+\theta_{L}$ both have the same periodicity.

Let us explain how all this can be interpreted in terms of conformal field theory. The gauging of the currents amounts to a generalization of the GKO cosetconstruction obtained by modding out by the left and right chiral current. Concretely this implies that one only keeps the fields which are primary with respect to the $U(1)_{R} \times U(1)_{L}$ current algebra. The difference between the axial and vector gauging is only in the assignment of the $U(1)$ quantum numbers $\left(q_{R}, q_{L}\right)$ that are carried by these primary fields. Following the above discussion we see that by reversing the signs of, say, all left charges $q_{L}$, while keeping the same right charges $q_{R}$, we go from one model to its dual. More precisely, for every primary field with charge $\left(q_{R}, q_{L}\right)$ there is a corresponding field in the dual model with charges $\left(q_{R},-q_{L}\right)$. All correlation functions are invariant under this operation, because conformal dimensions, OPE's, etc. depend only on quadratic combinations of the charges $q_{L}$. Thus we conclude that duality is truly a symmetry of the conformal field theory. 


\subsection{Some General Remarks on Duality}

One of the most interesting applications of the quotient construction and generalized duality is to the $2 \mathrm{~d}$ black hole CFT. For this we refer the reader to the literature 8 , 9, 10, [1]. It is not hard to think of other examples, but this we also leave to the reader. Here we will just comment on some general aspects of duality.

A first remark concerns the occurrence of singularities. When the isometry on the original manifold has a fixed point then by duality the dual manifold will have a singularity at the corresponding point. This can be illustrated with the somewhat trivial example of the $2 \mathrm{~d}$ plane. In polar coordinates the metric

$$
d s^{2}=d r^{2}+r^{2} d \theta^{2}
$$

becomes

$$
d s^{2}=d r^{2}+r^{-2} d \theta^{2}
$$

after duality. In addition there is a dilaton field $\Phi=\ln r^{2}$. So indeed the fixed point of the isometry, i.e., $r=0$, becomes a singular point after the duality transformation. The primary fields of the CFT on the $2 \mathrm{~d}$ plane all correspond to momentum modes. On the dual plane, however, there are, due to the singularity, no (normalizable) momentum modes; instead there are only winding modes. Duality tells us that, when we identify the winding numbers $m$ with the angular momentum quantum numbers $m$, corresponding dual fields have the same correlation functions. Thus, although the dual theory appears to be a singular theory, it actually behaves as the free theory on the plane.

For isometries corresponding to a compact $U(1)$, we can fix the periodicity of $\theta$ and $\tilde{\theta}$ to be $2 \pi$; different periods can be obtained by rescaling $g_{00}$ and the other components of the metric and $b$-field. Notice, however, that the period of $\tilde{\theta}$ must always be the reciprocal of that of $\theta$; otherwise, the two models are not fully equivalent but related via an orbifold construction. As a limiting case we can consider the duality transformation (2.7) for a non-compact isometry for which the corresponding $\theta$ coordinate can take any real value. We then find that the dual coordinate $\tilde{\theta}$ must have period zero, and so the dual manifold is actually an orbifold obtained by modding out the translations in $\tilde{\theta}$. For the confromal field theory this implies that the pure momentum states are removed and are again replaced by winding states.

Duality can also be applied to models with a lorentzian signature. Particularly 
interesting examples are backgrounds with a time-like killing vector $\mathbf{k}$, such as static black hole solutions. Here it may happen that in some region of space-time $\mathbf{k}$ becomes space-like. This means that at the boundary between the two regions, usually called the event horizon, $\mathbf{k}$ is a null vector. Since we have the relation

$$
g_{00}=\|\mathbf{k}\|^{2}
$$

we see that, as for fixed points, there will be a singularity in the dual space-time. Thus, the observation made in [10, 9] that the event horizon and the singularity of the $2 \mathrm{~d}$ black hole are interchanged by duality, is in fact much more general. Finally, we note that in principle the duality transformation (2.7) could change the signature of the manifold. This would be quite disturbing, but fortunately one can check that

$$
\operatorname{det} \tilde{g}_{i j}=\frac{1}{g_{00}^{2}} \operatorname{det} g_{i j},
$$

which shows that at least determinant of the metric does not change sign. We believe therefore that the signature of space-time is not altered by duality.

\section{Duality in $N=2$ Supersymmetric Sigma Models}

Somewhat surprisingly, the whole previous discussion can be lifted to $N=2$ superspace. First we introduce some notation.

\section{1. $\mathrm{N}=2$ Superspace}

In $N=2$ superspace, dynamics and kinematics are entwined in interesting ways. In addition to the choice of action, one has to choose representations of supersymmetry, i.e., superfields that obey various kinematical constraints (see for example 12]). Here we focus on two kinds of multiplets that are naturally dual to each other: chiral and twisted chiral multiplets [13]. (Other representations are possible [14]). The basic derivatives of $N=2$ superspace are complex left(right) moving spinor 
derivatives $D_{+}, D_{-}$that obey the algebra

$$
\left\{D_{+}, \bar{D}_{+}\right\}=\partial, \quad\left\{D_{-}, \bar{D}_{-}\right\}=\bar{\partial},
$$

all other (anti)commutators vanishing. The $N=2$ superspace action is written as 13

$$
S=\frac{1}{2 \pi} \int d^{2} z D_{+} D_{-} \bar{D}_{+} \bar{D}_{-} K(\Phi, \bar{\Phi}, \Lambda, \bar{\Lambda}),
$$

where $\Phi$ and $\Lambda$ are complex superfields obeying a chiral or twisted chiral constraint:

$$
\bar{D}_{ \pm} \Phi=0, \quad \bar{D}_{+} \Lambda=D_{-} \Lambda=0
$$

As a consequence of these constraints, the action changes only by a total derivative if we shift $K$ by functions $f(\Phi, \Lambda), g(\Phi, \bar{\Lambda})$, or the complex conjugates. The constraints can be explicitly solved by writing $\Phi, \Lambda$ in terms of an unconstrained complex superfield $\Psi$ :

$$
\Phi=i \bar{D}_{+} \bar{D}_{-} \Psi, \quad \Lambda=i \bar{D}_{+} D_{-} \Psi
$$

If $K$ is independent of the twisted chiral fields $\Lambda, \bar{\Lambda}$, then the manifold described is Kähler 15]; in the general case, the geometry has been described in 13.

\section{2. $\mathrm{N}=2$ Quotients AND DuAlity}

Because the geometry is restricted by $N=2$ supersymmetry, one can not take a usual quotient. In the Kähler case, the situation is well known [16, 17, 18], and one must perform a Kähler reduction. We describe the construction for the general case (4.2) in the appendix.

In practice, the $N=2$ quotient is performed very much in analogy with the bosonic quotient; we simply gauge the symmetry, not including a kinetic term for the gauge field, and eliminate the gauge field by its resulting equation of motion. Supersymmetry guarantees that the resulting quotient includes the moment map constraint described in the appendix. To do this, we need to review the superfield description of gauge fields, and introduce a (slight) modification of the known gauge 
multiplet, the twisted gauge multiplet that couples naturally to twisted chiral superfields. The usual gauge multiplet naturally gauges a vector symmetry, whereas the twisted multiplet naturally gauges an axial gauge symmetry. Both the usual gauge multiplet and the twisted gauge multiplet are described by a real unconstrained superfield $V$. They differ in that for the usual gauge multiplet the gauge transformation acts as a shift by the real part of a chiral superfield,

$$
\delta V=\Phi+\bar{\Phi}
$$

whereas for the twisted multiplet the gauge transformation acts as a shift by the real part of a twisted chiral superfield:

$$
\delta V=\Lambda+\bar{\Lambda}
$$

The corresponding super field-strengths also differ: for the usual gauge multiplet, the complex twisted chiral scalar $\bar{D}_{+} D_{-} V$ is gauge invariant, whereas for the twisted multiplet, the field-strength is chiral: $\bar{D}_{+} \bar{D}_{-} V$. (These field-strengths correspond to the physical scalars of the two-dimensional gauge multiplet). The only isometries that we can gauge without losing $N=2$ supersymmetry are ones that act holomorphically either on chiral superfields or on twisted chiral superfields. For the $U(1)$ case, the situation is particularly simple, and one can always choose coordinates such that $K$ is invariant and depends only on $\Phi+\bar{\Phi}$ or $\Lambda+\bar{\Lambda}$, depending on what kind of a gauging we want to perform. The "minimal" coupling prescription consists of merely substituting

$$
\Phi+\bar{\Phi} \rightarrow \Phi+\bar{\Phi}+V
$$

or

$$
\Lambda+\bar{\Lambda} \rightarrow \Lambda+\bar{\Lambda}+V
$$

However, that is not the whole story. There exist further gauge invariant terms (generically referred to as "Fayet-Iliopoulos terms" [19, 16]) that can and should be added. The original FI term consisted of just $V$; because the gauge parameters are constrained, up to a total superspace derivative, this is gauge invariant. In our case,

\footnotetext{
*One can also find "chiral" gauge multiplets that couple to the semichiral models of [14] in an obviously analogous way. Chris Hull has informed us that he has independently observed that such variant gauge multiplets can exist in two dimensions.
} 
when we have both chiral fields $(\Phi)$ and twisted chiral fields $(\Lambda)$, we can generalize this; for an arbitrary function $f$

$$
[f(\Lambda)+\bar{f}(\bar{\Lambda})] V
$$

is invariant (again, modulo total superspace derivatives) under chiral gauge transformations (4.5), and

$$
[f(\Phi)+\bar{f}(\bar{\Phi})] V
$$

is invariant under twisted chiral transformations (4.6). However, just as in the bosonic case, when we perform a duality transformation these terms can be absorbed by shifting the Lagrange multipliers: The condition that $V$ is pure gauge is imposed by constraining the appropriate field strengths, i.e., by including a term

$$
i\left(\Psi \bar{D}_{+} D_{-} V+\bar{\Psi} D_{+} \bar{D}_{-} V\right)
$$

for the usual gauge multiplet (4.5), or

$$
i\left(\Psi \bar{D}_{+} \bar{D}_{-} V+\bar{\Psi} D_{+} D_{-} V\right)
$$

for the twisted gauge multiplet (4.6). Here $\Psi$ is an unconstrained Lagrange multiplier field. Integrating by parts, these become

$$
(\Lambda+\bar{\Lambda}) V, \quad(\Phi+\bar{\Phi}) V
$$

respectively, where $\Lambda, \Phi$ are now constrained Lagrange multipliers. Clearly, (4.13) can be used to absorb (4.9,4.10).

\subsection{EXPLICIT SUPERSPACE DUALITY}

Concretely, consider a model with a superspace Lagrangian 13

$$
K=K\left(\Phi+\bar{\Phi}, X^{a}\right)
$$

where $X^{a}$ are "spectator" superfields that may be of any type (chiral, twisted chiral, etc.). The first order Lagrangian is

$$
K_{1}=K\left(\Phi+\bar{\Phi}+V, X^{a}\right)-(\Lambda+\bar{\Lambda}) V .
$$


Integrating out $\Lambda$ constrains $V$ to be pure gauge, and hence leads back to (4.14); integrating out $V$ gives

$$
\tilde{K}=K\left(V, X^{a}\right)-(\Lambda+\bar{\Lambda}) V
$$

where

$$
\frac{\partial K}{\partial V}=\Lambda+\bar{\Lambda}
$$

defines $V=V\left(\Lambda+\bar{\Lambda}, X^{a}\right)$. Thus $\tilde{K}$ is the Legendre transform of $K$ [20].

This discussion has been purely classical; quantum mechanically, one generally needs a dilaton as in the bosonic case. Curiously, in some circumstances (for example when the dual pair actually has an accidental $N=4$ supersymmetry), the dilaton contribution is automatically incorporated [6, 4, 5].

As in the bosonic case, we can learn about duality and conformal field theory quotients from the two-dimensional black hole. In [21, 22], the supersymmetric WZW model on $S U(2) \times U(1)$ was described in $N=2$ superspace in terms of one chiral and one twisted chiral superfield, and was shown to be dual to $[S U(2) / U(1)] \times$ $U(1)^{2}$.

We can follow the basic pattern and generalize this to arbitrary $N=2$ models with an appropriate isometry (as described above). We take a superspace Lagrangian'

$$
K=-\frac{1}{2}(\Lambda+\bar{\Lambda})^{2}+K_{0}\left(\Phi+\bar{\Phi}+\Lambda+\bar{\Lambda} ; X^{a}\right)
$$

where, as above, $X^{a}$ are spectator fields. Up to a total superspace derivative, this can be rewritten as

$$
K=\frac{1}{2}(\Phi+\bar{\Phi})^{2}+\hat{K}_{0}\left(\Phi+\bar{\Phi}+\Lambda+\bar{\Lambda} ; X^{a}\right)
$$

${ }^{\dagger}$ For $S U(2) \times U(1)[21,22]$,

$$
K=\int^{\frac{\Phi \bar{\Phi}}{\Lambda \Lambda}} \frac{d x}{x} \ln (1+x)-\frac{1}{2}(\ln \Lambda+\ln \bar{\Lambda})^{2} .
$$

where $\ln \Lambda$ plays the role of $\Lambda$ in the text here, etc. Up to total superspace derivatives, this can also be written as

$$
K=-\int^{\frac{\Lambda \bar{\Lambda}}{\Phi \Phi}} \frac{d x}{x} \ln (1+x)+\frac{1}{2}(\ln \Phi+\ln \bar{\Phi})^{2},
$$

These are the forms $(4.18)$ and 4.19 of the superspace action specialized to $S U(2) \times U(1)$. 
where

$$
\hat{K}_{0}(\Phi+\bar{\Phi}+\Lambda+\bar{\Lambda} ; X)=K_{0}-\frac{1}{2}(\Phi+\bar{\Phi}+\Lambda+\bar{\Lambda})^{2}
$$

As we saw above, quotients are inherently ambiguous; it is much safer to make a duality transformation. As in the bosonic case, we will find that after a duality transformation, we find the quotient model and a completely decoupled free field. We can make a duality transformation either with respect to $\Phi+\bar{\Phi}$ or $\Lambda+\bar{\Lambda}$; we use (4.18) or (4.19) as our starting point. Performing the duality transformation as described above (dropping total superspace derivatives), for the vector case we find

$$
\begin{aligned}
K^{+} & =-\frac{1}{2}(\Lambda+\bar{\Lambda})^{2}+K_{0}(V ; X)-(\tilde{\Lambda}+\tilde{\bar{\Lambda}})(V-\Phi-\bar{\Phi}-\Lambda-\bar{\Lambda}) \\
& =-\frac{1}{2}(\hat{\Lambda}+\hat{\bar{\Lambda}})^{2}+\frac{1}{2}(\tilde{\Lambda}+\tilde{\bar{\Lambda}})^{2}+K_{0}(V ; X)-(\tilde{\Lambda}+\tilde{\bar{\Lambda}}) V
\end{aligned}
$$

where $\hat{\Lambda}=\Lambda-\tilde{\Lambda}$ is a free field (and is decoupled), and $V$ is determined by

$$
\frac{\partial K^{+}}{\partial V}=\frac{\partial K_{0}}{\partial V}-\tilde{\Lambda}-\tilde{\bar{\Lambda}}=0
$$

For the axial gauging, we find

$$
\begin{aligned}
K^{-} & =\frac{1}{2}(\Phi+\bar{\Phi})^{2}+\hat{K}_{0}(V ; X)+(\tilde{\Phi}+\tilde{\bar{\Phi}})(V-\Phi-\bar{\Phi}-\Lambda-\bar{\Lambda}) \\
& =\frac{1}{2}(\hat{\Phi}+\hat{\bar{\Phi}})^{2}+K_{0}(V ; X)-\frac{1}{2}(V-\tilde{\Phi}-\tilde{\bar{\Phi}})^{2}
\end{aligned}
$$

where $\hat{\Phi}=\Phi-\tilde{\Phi}$ and $V$ is determined by

$$
\frac{\partial K^{-}}{\partial V}=\frac{\partial K_{0}}{\partial V}-V+\tilde{\Phi}+\tilde{\bar{\Phi}}=0
$$

After dropping the free field terms, the superspace actions $K^{+}(\tilde{\Lambda}+\tilde{\bar{\Lambda}} ; X)$ (4.21) and $K^{-}(\tilde{\Phi}+\tilde{\bar{\Phi}} ; X)(4.23)$ are indeed Legendre transforms of each other, and hence correspond to dual models. To show that any dual pair can be derived in this way, we note $(4.21)$ is a free field action for $\hat{\Lambda}$ combined with $\tilde{K}_{0}(\tilde{\Lambda}+\tilde{\bar{\Lambda}} ; X)+\frac{1}{2}(\tilde{\Lambda}+\tilde{\bar{\Lambda}})^{2}$, where $\tilde{K}_{0}$ is the Legendre transform of $K_{0}$. Hence if we are given some $K(\tilde{\Lambda}+\tilde{\bar{\Lambda}} ; X)$, and want to find $K_{0}(V ; X)$, we simply take the inverse Legendre transform of $K(\tilde{\Lambda}+$ $\tilde{\bar{\Lambda}} ; X)-\frac{1}{2}(\tilde{\Lambda}+\tilde{\bar{\Lambda}})^{2}$. 


\section{4. $N=2$ Duality in Components}

Though the $N=2$ construction is in many ways analogous to the bosonic construction, the relation can be made more precise by working out the component form. As the basic superspace technology is readily available (see for example [12, 13, 17]), we give only the relevant results. In particular, since we are interested in comparing to the bosonic expressions of the previous sections, we drop all fermionic terms.

To proceed, we must specify the constraints on the spectator fields $X^{a}$; we choose to divide them into two sets, chiral superfields $X^{i}$ and twisted chiral superfields $X^{r}$. We further introduce the following notation:

$$
\rho=\operatorname{Re}(\Phi+\Lambda), \quad \varphi=\operatorname{Re}(\Phi-\Lambda), \quad \theta_{R}=\operatorname{Im}(\Phi+\Lambda), \quad \theta_{L}=\operatorname{Im}(\Phi-\Lambda)
$$

for the leading bosonic components of the superfields $\Phi, \Lambda$, and

$$
x^{a}=\operatorname{Re} X^{a}, \quad y^{a}=\operatorname{Im} X^{a}
$$

for the leading bosonic components of the "spectator fields" $X$. It is also useful to express the answer not in terms of $K_{0}$ or $\hat{K}_{0}$ above (see (4.20), but in terms of the average $\bar{K}=\frac{1}{2}\left(K_{0}+\hat{K}_{0}\right)$.

The bosonic component action that results from (4.18,4.19) is then

$$
\begin{gathered}
S=\frac{1}{2 \pi} \int d^{2} z\left[\partial \theta_{L} \bar{\partial} \theta_{L}+\partial \theta_{R} \bar{\partial} \theta_{R}+4 \bar{K}^{\prime \prime} \bar{\partial} \theta_{L} \partial \theta_{R}+G_{a}^{R} \partial \theta_{R} \bar{\partial} x^{a}-G_{a}^{L} \partial x^{a} \bar{\partial} \theta_{L}\right. \\
\left.+\partial\left(\varphi+\bar{K}^{\prime}\right) \bar{\partial}\left(\varphi+\bar{K}^{\prime}\right)\right]+S[\rho, x, y]
\end{gathered}
$$

where $\bar{K}^{\prime} \equiv \frac{\partial \bar{K}(\rho ; x, y)}{\partial \rho}$, etc., and

$$
\begin{array}{llll}
G_{x^{i}}^{R}=2 \bar{K}_{x^{i}}^{\prime} & G_{x^{r}}^{R}=-2 \bar{K}_{x^{r}}^{\prime} & G_{y^{i}}^{R}=-2 \bar{K}_{y^{i}}^{\prime} & G_{y^{r}}^{R}=2 \bar{K}_{y^{r}}^{\prime} \\
G_{x^{i}}^{L}=2 \bar{K}_{x^{i}}^{\prime} & G_{x^{r}}^{L}=2 \bar{K}_{x^{r}}^{\prime} & G_{y^{i}}^{L}=-2 \bar{K}_{y^{i}}^{\prime} & G_{y^{r}}^{L}=-2 \bar{K}_{y^{r}}^{\prime}
\end{array}
$$

This is precisely of the form (3.5) with an extra free field $\varphi+\bar{K}^{\prime}$. This free field that is present before duality explains why $N=2$ duality decouples a complex field.

Acknowledgements: We would like to thank P. Ginsparg, A. Giveon, E. Witten for discussions and M. Best for help with $\mathrm{T}_{\mathrm{E}} \mathrm{X}$. EV wishes to thank the Aspen Center 
for Physics, where part of this work was done. The work of MR is supported in part by NSF grant No. PHY 91-08054 and by the John Simon Guggenheim Foundation, and that of EV by the W.M. Keck Foundation.

\section{Appendix: The geometry of $N=2$ SUPERSymmetric QuOtients}

We begin by reviewing the geometry of $N=2$ models as described in [13]. In general, $N=2$ supersymmetric models can be constructed on complex manifolds with two complex structures $\mathcal{J}^{ \pm}$that are both compatible with the metric $g$, and that are preserved by connections $\Gamma^{ \pm}=\Gamma^{0} \pm T:\left(\partial+\Gamma^{ \pm}\right) \mathcal{J}^{ \pm}=0$. Here $\Gamma^{0}$ is the Levi-Civita connection constructed just out of the metric and $T$ is the torsion $T=\frac{3}{2} d b$ as in the text. It follows that $\omega_{i j}^{ \pm} \equiv g_{i k} \mathcal{J}^{ \pm k}{ }_{j}$ is in general not closed but obeys $\partial^{ \pm} \bar{\partial}^{ \pm} \omega^{ \pm}=0$. If the torsion vanishes, then $\mathcal{J}^{+}=\mathcal{J}^{-}$, the manifold is Kähler and $d \omega=0$. Quotients will preserve $N=2$ supersymmetry if and only if they preserve these geometric properties.

In the Kähler case, the $N=2$ quotient can be performed for any isometry that preserves the Kähler form. The procedure is well known: since $\omega$ is a (closed) symplectic form, the equation $\mathcal{L}_{\mathbf{k}} \omega=0$ can be integrated to

$$
\omega_{i j} \mathbf{k}^{j}=\partial_{i} \mu,
$$

which defines the moment map $\mu$ up to an integration constant. The Kähler quotient is then simply the ordinary quotient on the submanifold $\mu=0$.

A generalization to the more general $N=2$ geometry exists when the following conditions are satisfied (we have not determined which of them are independent and which are implied by a subset): There must be a family of submanifolds on which the commutator $\left[\mathcal{J}^{+}, \mathcal{J}^{-}\right]$of the two complex structures vanishes. Each such submanifold further admits two families of smaller submanifolds on which $\mathcal{J}^{+}=$ $\pm \mathcal{J}^{-}$; any of these smallest submanifolds is Kähler, where the Kähler form is the restriction of $\omega^{ \pm}$.

The isometry must preserve both complex structures as well as the torsion, i.e., $\mathcal{L}_{\mathrm{k}} \mathcal{J}^{ \pm}=\mathcal{L}_{\mathrm{k}} T=0$. Furthermore, the isometry must be tangent to one of the two families defined above; without loss of generality, we assume

$$
\mathcal{J}^{+i}{ }_{j} \mathbf{k}^{j}=\mathcal{J}^{-i}{ }_{j} \mathbf{k}^{j} .
$$

We now construct the moment map $\mu$ on this Kähler submanifold. The integration 
constants are allowed to depend on the coordinates of the other family of submanifolds as long as

$$
\left(\partial^{+}-\partial^{-}\right)\left(\bar{\partial}^{+}-\bar{\partial}^{-}\right) \mu=0 .
$$

The quotient is again taken on the submanifold $\mu=0$. This generalizes the usual moment map construction.

\section{References}

[1] K. Kikkawa and M. Yamasaki, Phys. Lett. 149B (1984) 357.

[2] W. Siegel, Phys. Lett. 134B (1984) 318.

[3] B.E. Fridling and A. Jevicki, Phys. Lett. 134B (1984) 70.

[4] T. Buscher, Phys. Lett. 194B (1987) 59.

[5] T. Buscher, Phys. Lett. 201B (1988) 466.

[6] T. Buscher, Phys. Lett. 159B (1985) 127.

[7] A.A. Tseytlin, Mod. Phys. Lett. A6 (1991) 1721.

[8] E. Witten, Phys. Rev. D44 (1991) 314.

[9] R. Dijkgraaf, E. Verlinde, and H. Verlinde, "String Propagation in a Black Hole Geometry", IAS preprint IASSNS-HEP-91/22.

[10] A. Giveon, "Target Space Duality and Stringy Black Holes", LBL preprint no. LBL-30671 (1991).

[11] E.B. Kiritsis, "Duality in Gauged WZW Models", LBL preprint no. LBL-30747 (1991).

[12] S.J. Gates, M.T. Grisaru, M. Roček, and W. Siegel, Superspace, Benjamin Cummings, Reading USA (1983).

[13] S.J. Gates, C.M. Hull, and M. Roček, Nucl. Phys. B248 (1984) 157.

[14] T. Buscher, U. Lindström, and M. Roček, Phys. Lett. 202B (1988) 94.

[15] B. Zumino, Phys. Lett. 87B (1979) 203. 
[16] J. Bagger and E. Witten, Phys. Lett. 118B (1982) 103.

[17] N.J. Hitchin, A. Karlhede, U. Lindström, and M. Roček, Commun. Math. Phys. 108 (1987) 535.

[18] C.M. Hull, A. Karlhede, U. Lindström, and M. Roček, Nucl. Phys. B266 (1986) 1.

[19] P. Fayet and J. Iliopoulos, Phys. Lett. 51B (1974) 461.

[20] U. Lindström and M. Roček, Nucl. Phys. B222 (1983) 285.

[21] M. Roček, K. Schoutens, and A. Sevrin, Phys. Lett. 265B (1991) 303.

[22] C. Ahn, M. Roček, K. Schoutens, and A. Sevrin, "Superspace WZW models and Black Holes" Stony Brook preprint ITP-SB-91-49. 